\title{
PERSONALIDAD Y PERCEPCIÓN DE AUTOEFICACIA: INFLUENCIA SOBRE EL BIENESTAR Y EL AFRONTAMIENTO DE LOS PROBLEMAS DE SALUD
}

\author{
BEATRIZ RUEDA y ANA M. PÉREZ-GARCÍA \\ Universidad Nacional de Educación a Distancia
}

(Aceptado en septiembre de 2004)

\begin{abstract}
Esta investigación examinó el papel que desempeñan la personalidad y determinados factores psicosociales en relación con criterios de salud (bienestar, satisfacción y conductas preventivas), y el afrontamiento ante los problemas de salud. Ciento setenta y tres participantes completaron medidas de los Cinco Factores, Percepción de Autoeficacia Generalizada (AEG) y Competencia Percibida en Salud (CPS), junto con criterios de salud y afrontamiento. Los resultados mostraron que los rasgos se conectaron más con la satisfacción, mientras que AEG y CPS se vincularon más estrechamente con las conductas preventivas y el bienestar, respectivamente. Asimismo los rasgos dieron cuenta de una mayor varianza del afrontamiento instrumental y emocional, en comparación con AEG y CPS. Sin embargo CPS fue el principal predictor de estos dos tipos de afrontamiento. Estos resultados sugieren que la consideración conjunta de las dimensiones de personalidad y los factores psicosociales permite clarificar su utilidad predictiva en el campo de la salud.
\end{abstract}

Palabras clave: Cinco Factores, autoeficacia, competencia percibida, bienestar, afrontamiento.

\section{Personality and Self-Efficacy: Influence on well-being and coping with health problems}

This investigation examined the role that personality and psychosocial factors play in relation to health outcomes (well-being, satisfaction and preventive behaviours), and coping with health problems. One hundred and seventy three participants completed measures of the Big Factors, Generalized Self-Efficacy (GSE) and Perceived Health Competence (PHC), along with health outcomes and coping. Results showed that traits were more connected to satisfaction, whereas GSE and PHC were linked most strongly with preventive behaviours and well-being, respectively. Furthermore traits accounted for a higher variance in instrumental and emotional coping, in comparison to GSE and PHC. Nevertheless PHC was the main predictor of these two kinds of coping. These findings suggest that the consideration of personality dimensions and psychosocial factors in tandem allows to clarify their predictive utility in the health domain.

Key words: Big Five, self-efficacy, perceived competence, well-being, coping.

\section{INTRODUCCIÓN}

La idea de que las características de personalidad pueden desempeñar una notable influencia sobre la forma de afrontar el estrés, los niveles de bienestar y la pre-

Correspondencia: Beatriz Rueda, Universidad Nacional de Educación a Distancia, Facultad de Psicología. Departamento de Psicología de la Personalidad, Evaluación y Tratamiento Psicológicos, C/ Juan del Rosal, 10, 28040 Madrid. Teléfono: 91398 6281, Fax: 91398 6298, correo-e: brueda@psi.uned.es vención de la enfermedad, constituye un área de gran interés en la que han convergido numerosas investigaciones (Bermúdez, 1999; DeNeve y Cooper, 1998; Smith y Williams, 1992; Van Heck, 1997).

Por otro lado, a pesar de que aún no se han alcanzado conclusiones firmes acerca de los mecanismos específicos mediante los que la personalidad afecta a la salud, el tipo de afrontamiento empleado ante los acontecimientos estresantes 
parece constituir un mecanismo relevante (Chang, 1998; Rueda, Pérez-García y Bermúdez, 2003).

A la hora de abordar el estudio de la personalidad en el contexto de la salud, numerosos estudios (Bermúdez, 1999; Booth-Kewley y Vikers, 1994; Marshall et al., 1994; Van Heck, 1997) coinciden en señalar que es necesario tener en cuenta tanto los aspectos estructurales de la personalidad, como las unidades intermedias relacionadas con los procesos que originan la conducta.

Tomando como punto de referencia la vertiente más estructural de la personalidad, un modelo que ha recibido una atención creciente, por su adecuación para representarla de manera comprehensiva (Marshall et al., 1994), es el modelo de los Cinco Factores (Norman, 1963; McCrae y Costa, 1985).

De acuerdo con este paradigma, la personalidad estaría configurada en cinco dimensiones amplias, que implicarían tendencias estables y consistentes de respuesta (John, 1990). Estas dimensiones son la Extraversión, que implica aspectos como la asertividad y el dinamismo; la Afabilidad, que supone la capacidad de ser compasivo y confiado; el Tesón, el cual incluye la tendencia a la organización y la eficiencia; el Neuroticismo, que equivale a la inestabilidad emocional y la experiencia de estados emocionales negativos; y la Apertura a la Experiencia, que refleja el mantenimiento de valores e ideas no convencionales, y la amplitud de intereses. McCrae y Costa $(1987,1997)$ han obtenido evidencia respecto de estos factores empleando muestras de diferentes nacionalidades, e instrumentos de medición diversos.

De otro lado, la comprensión de la personalidad a partir de unidades de nivel intermedio, organizadas en procesos de mayor dinamismo (Cantor, 1990; Van Heck, 1997), puede ampliar el conocimiento acerca de las conexiones entre la personalidad y la salud, al contemplar la influencia del contexto donde ocurre la conducta.

En particular, la percepción de autoeficacia constituye un tipo de proceso con una clara influencia sobre la realización de la conducta (Bandura, 1989). En los términos propuestos por Bandura (1977; 1997), la expectativa de autoeficacia hace referencia a la estimación personal de que se es capaz de llevar a cabo una conducta específica. Otros autores (Baessler y Schwarzer, 1996; Schwarzer, 1993) han planteado esta creencia de forma más global, refiriéndola a la confianza que se tiene en la capacidad para solventar las situaciones de estrés y considerándola como una expectativa de autoeficacia generalizada (AEG). Finalmente, desde una óptica más intermedia, Wallston y colaboradores (Smith, Wallston y Smith, 1995) han sugerido la competencia percibida en salud (CPS), designando con ella el nivel de autoeficacia que se percibe en relación con los problemas y asuntos relativos a la salud.

\section{Personalidad, bienestar y conductas de salud}

Uno de los resultados más consistentemente observado y replicado cuando se consideran las relaciones entre los rasgos de personalidad y el bienestar subjetivo, es la asociación positiva entre la Extraversión y el bienestar, y la conexión negativa entre este criterio y el Neuroticismo (DeNeve y Cooper, 1998; Diener, 1996; Emmons y Diener, 1985; Pavot, Diener y Fujita, 1990). La Afabilidad y el Tesón también parecen contribuir a explicar positivamente la satisfacción vital (McCrae y Costa, 1991; Watson y Clark, 1992), bien porque faciliten el establecimiento de redes de apoyo, o porque promuevan la consecución de las metas.

Con respecto a la AEG (Bonetti et al., 2001; Sanz y Villamarín, 1999) y la CPS 
(Pastor et al., 1999; Smith et al., 1995), se ha evidenciado que ambas expectativas se relacionan con una disminución en la presencia de síntomas y de respuestas emocionales negativas.

Puesto que diferentes estudios han mostrado que los rasgos de personalidad son adecuados para predecir criterios globales de salud (Judge, Erez, Bono y Thoresen, 2002; Prenda y Lachman, 2001; Wasylkiw y Fekken, 2002), a la hora de analizar la capacidad explicativa de la AEG y la CPS junto con la de los Cinco Factores, se puede esperar que los rasgos den cuenta de una mayor variabilidad en relación con el bienestar y la satisfacción, ya que su nivel de generalidad estará más ajustado a estos criterios.

En lo que a las conductas de salud se refiere, diferentes investigaciones han señalado que los rasgos de afabilidad y tesón se asocian positivamente con la realización de acciones promotoras de la salud (Lemos-Giráldez y Fidalgo-Aliste, 1997; Vollrath, Knoch y Cassano, 1999), mientras que el Neuroticismo presenta una mayor conexión con conductas de riesgo (Vollrath y Torgersen, 2002). A pesar de ello, la validez predictiva que tienen estos factores parece ser moderada, ya que en estos estudios el porcentaje de varianza explicado de las conductas de salud no excedió en ningún caso el $30 \%$.

A este respecto, la mayor parte de las teorías actuales sobre conductas de prevención apoyan la inclusión de la AEG como uno de sus principales determinantes (Schwarzer 1999; Wallston, 1992; Weinstein, 1993). De igual modo, la CPS también se ha asociado con el mantenimiento de un estilo de vida más saludable (Gebhardt, van der Doef y Paul, 2001; Smith et al., 1995).

Si bien hasta la fecha la investigación acerca de la contribución conjunta de los rasgos y las expectativas de autoeficacia con respecto a la variabilidad de las con- ductas de prevención es escasa, los resultados tienden a avalar la superioridad en la utilidad predictiva de las creencias de autoeficacia (Conner y Abraham, 2001; Marks y Lutgendorf, 1999).

\section{Personalidad y afrontamiento}

El estudio del afrontamiento dentro del contexto de la personalidad ha sido abordado desde dos enfoques diferentes.

Por un lado, se ha recurrido a las grandes taxonomías de personalidad para analizar los aspectos estructurales del afrontamiento (Ferguson, 2001; McCrae y Costa, 1986). De este modo el afrontamiento ha sido considerado como un estilo de respuesta estable y consistente que se pone en marcha ante el estrés.

El segundo enfoque que se ha aplicado ha consistido en examinar el afrontamiento asociándolo con características más dinámicas de personalidad (Watson y Hubbard, 1996). En este sentido, la percepción de autoeficacia ha sido contemplada como una variable antecedente de las respuestas de afrontamiento (Carver, Scheier y Weintraub, 1989; Folkman, 1984; Lazarus y Folkman, 1987).

En particular se ha constatado que, tanto la AEG como la CPS, promueven la utilización de un tipo de afrontamiento centrado en la modificación de las circunstancias estresantes (Schwarzer, 1993; Sanjuán, Pérez-García y Bermúdez, 2000), y en la disminución de las estrategias pasivas o de evitación (Smith et al., 1995).

Dentro del ámbito de la salud, Endler y colaboradores (Endler, Courbasson y Fillion, 1998; Endler, Parker y Summerfeldt, 1998) han propuesto una categorización del afrontamiento de nivel intermedio, destinada a clasificar las estrategias de afrontamiento que se ponen en marcha ante los problemas de salud. De acuerdo con esta perspectiva, 
existirian cuatro tipos de reacciones ante los diferentes problemas de salud: el afrontamiento instrumental, dirigido a la búsqueda de asistencia médica e información; el afrontamiento paliativo, el cual incluye una serie de acciones para tratar de amortiguar los efectos negativos del problema; el afrontamiento emocional, que atiende a los aspectos emocionales negativos; $y$, por último, el afrontamiento de distracción, con el que se intenta evitar pensar en el problema o buscar la compañía de otras personas.

La investigación sistemática de las variables de personalidad que predicen este tipo de afrontamiento, y del tipo de contribución que cada una de ellas puede aportar, se encuentra aún poco desarrollada. Tan sólo el estudio de Rueda (2003) ha indicado que la competencia percibida en salud correlacionaba positivamente con el afrontamiento instrumental de la salud, y negativamente con el afrontamiento emocional. En esta investigación, sin embargo, no fueron incluidas las dimensiones básicas de personalidad. Es por ello que, la asociación de estas respuestas de afrontamiento con los Cinco Factores, por un lado, y con la autoeficacia generalizada y la competencia percibida en salud por otro, requiere una mayor profundización.

\section{Objetivos e hipótesis}

El primer objetivo de este trabajo ha sido el análisis comprehensivo de los Cinco Factores, la AEG y la CPS, en relación con la predicción de determinados criterios de salud, como son el bienestar subjetivo, la satisfacción personal y las conductas de prevención. En este sentido se espera que las dimensiones básicas de la personalidad contribuyan, en mayor medida que las expectativas de autoeficacia, a explicar el bienestar y la satisfacción. Las variables de nivel inter- medio, como la creencia de autoeficacia y la competencia percibida en salud, por su parte, presentarán una mayor utilidad a la hora de explicar las conductas de prevención.

El segundo objetivo de la investigación ha consistido en explorar y comparar la capacidad predictora de las dimensiones básicas de la personalidad y las dos creencias de autoeficacia (generalizada y centrada en la salud), con respecto a las estrategias de afrontamiento ante la enfermedad.

\section{MÉTODO}

\section{Participantes}

La muestra estuvo compuesta por 173 participantes ( $76 \%$ mujeres y $24 \%$ varones), siendo la edad media de 33 años y oscilando el rango entre los 17 y los 67 años. Todos los participantes fueron estudiantes de la UNED de los primeros cursos de psicología.

En un primer momento de la investigación se entregó en mano a los participantes un cuestionario que incluía las variables concernientes a las dimensiones de personalidad, la percepción de autoeficacia, el bienestar y la satisfacción general. Una vez completado el cuestionario, los participantes debían devolverlo enviándolo por correo.

Al cabo de los tres meses se volvió a remitir un nuevo cuestionario incluyendo, entre otras variables, las relativas a la salud, es decir, la competencia percibida en salud, el afrontamiento de los problemas de salud y las conductas de prevención. En la segunda parte del estudio continuaron 110 estudiantes (79\% mujeres y $21 \%$ varones). La edad media fue de 33 años, y el rango estuvo comprendido entre los 18 y los 67 años.

La recogida de datos se llevó a cabo en dos fases para evitar el cansancio de los 
participantes en la cumplimentación de las pruebas, y el posible sesgo en su respuesta a las mismas.

En cuanto a las diferencias de género, las mujeres puntuaron significativamente más alto en Afabilidad $\left(M=87,73 ; t_{\text {(ass }}=\right.$ $4,41, p<0,008)$, el afrontamiento centrado en la distracción $\left(M=25,02 ; t_{\text {(10) }}=2,71\right.$, $p<0,008)$, y prevencion $\left(M=86,57 ; t_{(10)}=\right.$ $2,44, p<0,02$ ), en comparación con los hombres (Afabilidad: $M=79,95$; distraccion: $M=22$; y prevención: $M=83,31$ ).

Asimismo, entre los participantes que continuaron en la investigación y los que la abandonaron, sólo se encontraron diferencias significativas en el rasgo de Afabilidad $\left.\left(t_{1+1}\right)=2,23, p<0,05\right)$.

\section{Instrumentos de medida}

Cinco Factores: Las cinco dimensiones básicas de personalidad se midieron a través del BFQ (Caprara, Barbaranelli y Borgogni 1995; adaptación española de Bermúdez). Este instrumento se compone de 132 ítems ante los que se debe valorar el grado de verdad o falsedad de cada enunciado en relación con la manera de pensar y sentir de uno mismo. El cuestionario comprende 5 escalas que se refieren a los rasgos de Energía, Afabilidad, Tesón, Estabilidad Emocional y Apertura Mental; y 10 subescalas que representan las 10 facetas de los rasgos (Dinamismo y Dominancia para Energía; Cooperación y Cordialidad para Afabilidad; Escrupulosidad y Perseverancia para Tesón; Control Emocional y Control de Impulsos para Estabilidad Emocional; y Apertura a la Cultura y Apertura a la Experiencia para Apertura Mental). Asimismo el BFQ incluye una escala de deseabilidad social. En el presente estudio sólo se consideraron las medidas referidas a los Cinco Factores, para predecir los criterios de salud a partir de predictores con un mayor nivel de gene- ralización. El BFQ ha demostrado tener una adecuada validez transcultural, oscilando los coeficientes de congruencia desde 0,94 hasta 0,99 (Barbaranelli, y Caprara, 2002). En otros estudios se han obtenido coeficientes de fiabilidad elevados, situándose entre 0,94 y 0,99 (Barbaranelli, y Caprara, 2002; Caprara, Barbaranelli, Borgogni y Perugini, 1993).

Percepción de Autoeficacia Generalizada (Schwarzer, 1993): Esta escala, compuesta de 10 ítems, mide la expectativa que se tiene acerca de la capacidad para afrontar adecuadamente cualquier situación problemática. El formato de respuesta oscila entre 1 (Nada) y 5 (Mucho). La prueba ha mostrado una consistencia interna alta en muestras tanto de pacientes (Pastor et al., 1999) como de estudiantes (Sanjuán et al., 2000). En el presente trabajo el índice de fiabilidad fue de 0,90.

Competencia Percibida en Salud (Smith et al., 1995): Este instrumento mide la creencia de competencia que se tiene sobre el manejo de la salud. Se compone de 8 items, con un formato de respuesta tipo likert que va desde 1 (Nada) hasta 5 (Mucho). Investigaciones previas han obtenido un coeficiente a moderadamento alto, comprendido entre 0,60 y 0,90 (Pastor et al., 1999; Smith et al., 1995). En nuestro estudio el coeficiente de fiabilidad fue de 0,86 .

Estrategias de Afrontamiento de los problemas de Salud (Endler y Parker, 1999): Esta prueba contiene 32 ítems que evalúan cuatro tipos de estrategias de afrontamiento (8 ítems por escala) ante los problemas de salud: el afrontamiento instrumental, el afrontamiento emocional, el paliativo y el centrado en la distracción. El formato de respuesta es tipo Likert con 5 puntos (donde 1 = Nada y 5 $=$ Mucho). La consistencia interna del cuestionario ha demostrado ser alta, 
situándose por encima de 0,70 (Endler, Kocovski y Macrodimitris, 2001; Endler, Parker y Summerfeldt, 1998). En la presente investigación los coeficientes de fiabilidad fueron 0,77 para el afrontamiento instrumental; 0,76 para el afrontamiento emocional; 0,69 para el paliativo; y 0,69 para el centrado en la distracción.

Calidad de vida: Para medir las dimensiones de bienestar y satisfacción personal, se emplearon las dos subescalas del Cuestionario de Calidad de Vida (Ruiz y Baca, 1993). Este cuestionario se compone de 35 ítems, organizados en cuatro dominios: apoyo social, bienestar físico y psicológico, satisfacción personal y tiempo libre. El formato de respuesta es tipo Likert con una escala de cinco puntos (donde $1=$ Nada y $\mathbf{5}=$ Mucho). La consistencia interna de las subescalas de bienestar y de satisfacción fue, en ambos casos, de 0,86.

Conductas de Prevención: Para medir esta variable se utilizó un inventario con 24 conductas de salud (p. ej. consumo de alimentos ricos en fibra, cuidado del peso, control de la tensión, etc.), ante las que los participantes debían indicar con qué frecuencia las llevaban a cabo. El formato de respuesta osciló desde 1 (Nunca) hasta 5 (Siempre). A partir de la puntuación dada a cada ítem se obtuvo un índice global de conductas de prevención. De esta manera una puntuación alta significaba una mayor frecuencia en conductas de salud. El coeficiente alpha de la presente escala fue de 0,81 .

\section{RESULTADOS}

Relaciones entre las variables de personalidad y los criterios de salud

En primer lugar se obtuvieron los estadísticos descriptivos (medias, desviaciones típicas y rango) relativos a las variables predictoras y los criterios. Estos datos se presentan en la Tabla 1.

A continuación se hallaron las correlaciones de los Cinco Factores, la AEG y la CPS con los criterios de salud. Como puede observarse en la Tabla 2, los rasgos de personalidad, a excepción del Tesón, correlacionaron positivamente con el bienestar y la satisfacción. Con las conductas de prevención, sin embargo, no se obtuvo ninguna correlación significativa. Tan sólo la Afabilidad se asoció con esta variable, aunque tal relación alcanzó un nivel de significación marginal.

Tabla 1. Medias, desviaciones típicas y rango de las variables de personalidad, los criterios de salud y las estrategias de afrontamiento

\begin{tabular}{lccc}
\hline Variables & Media & Desv. Típica & Rango \\
\hline Energía & 74,99 & 10,85 & $53-109$ \\
Afabilidad & 86,53 & 9,21 & $62-116$ \\
Tesón & 83,94 & 10,62 & $61-111$ \\
Estabilidad Emocional & 67,38 & 13,22 & $33-99$ \\
Apertura Mental & 87,91 & 9,17 & $65-108$ \\
Autoeficacia Generalizada & 36,17 & 5,60 & $18-50$ \\
Competencia P. Salud & 31,16 & 4,15 & $20-40$ \\
Bienestar & 27,90 & 4,98 & $10-35$ \\
Satisfacción & 46,94 & 7,41 & $26-65$ \\
Conductas Prevención & 85,19 & 11,82 & $56-108$ \\
Afrontamiento Instrumental & 31,17 & 4,42 & $15-40$ \\
Afrontamiento Emocional & 22,40 & 5,29 & $12-35$ \\
Afrontamiento Paliativo & 25,90 & 4,12 & $11-36$ \\
Afrontamiento Distracción & 24,39 & 4,89 & $12-33$ \\
\hline
\end{tabular}


Tabla 2. Correlaciones entre las variables de personalidad y los criterios de salud

\begin{tabular}{|c|c|c|c|}
\hline \multirow[b]{2}{*}{ Variables } & \multicolumn{3}{|c|}{ Criterios de salud } \\
\hline & Bienestar & Satisfacción & Conductas de Prevención \\
\hline Energía & $0,25^{*}$ & $0,39^{\cdots \cdots}$ & 0,15 \\
\hline Afabilidad & $0,21^{*}$ & $0,27^{\cdots \cdots}$ & $0,18+$ \\
\hline Tesón & 0,02 & 0,10 & 0,16 \\
\hline Estabilidad Emocional & $0,32 \cdots$ & $0,27^{* \cdots}$ & 0,07 \\
\hline Apertura Mental & $0,25 *$ & $0,26{ }^{* *}$ & 0,15 \\
\hline Autoeficacia Generalizada & $0,37^{* * *}$ & $0,58^{* * *}$ & $0,36^{\cdots *}$ \\
\hline Competencia P. Salud & $0,63^{* * *}$ & $0,49^{* * *}$ & $0,39^{\cdots \cdots}$ \\
\hline
\end{tabular}

"* $p<0,001 ; "$ " $p<0,01 ;+p=0,06$.

Por otra parte, la AEG y la CPS correlacionaron positivamente con el bienestar y la satisfacción. Estas correlaciones fueron superiores a las halladas con los rasgos de personalidad, poniendo de manifiesto que el mantenimiento de estas dos creencias puede tener una mayor influencia sobre el bienestar y la satisfacción personal, que la que pueden ejercer las características más estructurales de la personalidad. Asimismo, como se esperaba, las correlaciones de la AEG y la CPS con las conductas de prevención fueron positivas y significativas. De esta manera, el sentirse capaz de manejar eficazmente diferentes situaciones, o aquéllas específicamente relacionadas con la salud, favorece la realización de conductas promotoras de la salud.

En cuanto a las estrategias de afrontamiento de los problemas de salud (ver Tabla 3), tanto los rasgos de personalidad como la AEG y la CPS correlacionaron negativamente con el afrontamiento emo- cional, siendo estas correlaciones ligeramente más altas en el caso de las expectativas de autoeficacia (general y de salud). Las correlaciones con la Afabilidad y el Tesón no fueron significativas. El afrontamiento instrumental, por su parte, correlacionó positivamente con el Tesón y la Apertura Mental, así como con la AEG y la CPS. A tenor de estos resultados podría deducirse que, mientras que los rasgos de Energía, Tesón y Estabilidad Emocional parecen vincularse con un determinado tipo de estrategia de afrontamiento, la Apertura Mental y las dos creencias de autoeficacia tienden a modular el uso del afrontamiento instrumental (relación positiva) y el emocional (relación negativa).

Con respecto al afrontamiento paliativo y al centrado en la distracción, apenas se encontró alguna asociación significativa con las características de personalidad. Solamente la Afabilidad se asoció positivamente con el afrontamiento dirigido a la distracción, indicando así que

Tabla 3. Correlaciones entre las variables de personalidad y las estrategias de afrontamiento

\begin{tabular}{|c|c|c|c|c|}
\hline \multirow[b]{2}{*}{ Variables } & \multicolumn{4}{|c|}{ Afrontamiento } \\
\hline & Instrumental & Emocional & Paliativo & Distracción \\
\hline Energía & 0,00 & $-0,22^{\star}$ & $-0,00$ & $0,17+$ \\
\hline Afabilidad & 0,09 & $-0,08$ & 0,13 & $0,21^{*}$ \\
\hline Tesón & $0,36^{* * *}$ & $-0,06$ & 0,08 & 0,11 \\
\hline Estabilidad Emocional & 0,14 & $-0,34^{* *}$ & $-0,06$ & $-0,06$ \\
\hline Apertura Mental & $0,26^{* *}$ & $-0,25^{* *}$ & 0,10 & 0,13 \\
\hline Autoeficacia Generalizada & $0,23^{*}$ & $-0,40^{\cdots *}$ & 0.03 & 0,16 \\
\hline Competencia P0, Salud & $0,22^{*}$ & $-0,39^{* * *}$ & $0,17+$ & 0,17 \\
\hline
\end{tabular}

$\cdots p<0,001 ; " p<0,01 ; " p<0,05 ;+p=0,07$. 
las personas afables recurren más a su entorno social cuando se encuentran con problemas de salud.

Predictores de los criterios de salud y las estrategias de afrontamiento

Para determinar en qué medida los rasgos de personalidad, así como las expectativas de nivel intermedio evaluadas, daban cuenta de las diferencias en bie- nestar, satisfacción y prevención, se llevaron a cabo análisis de regresión jerárquico. En relación con el bienestar y la satisfacción, se introdujeron, en primer lugar, los cinco factores, a continuación la AEG y, finalmente, la CPS. En relación con las conductas de prevención se controló además el efecto del género, ya que previamente se habían encontrado diferencias en las conductas de prevención en función de esta variable.

Tabla 4. Análisis de regresión jerárquicos prediciendo bienestar, satisfacción y conductas de prevención

\begin{tabular}{|c|c|c|c|c|c|}
\hline Criterios & $\beta$ & $R^{2}$ & $\Delta R^{2}$ & $g l$ & $F$ \\
\hline \multicolumn{6}{|l|}{ Bienestar } \\
\hline \multicolumn{6}{|l|}{ Paso1 } \\
\hline Energía & $0,18^{*}$ & 0,20 & 0,20 & 5,104 & $50,04^{\cdots}$ \\
\hline Tesón & $-0,05$ & & & & \\
\hline Estabilidad Emocional & 0,15 & & & & \\
\hline Afabilidad & 0,02 & & & & \\
\hline Apertura Mental & 0,05 & & & & \\
\hline \multicolumn{6}{|l|}{ Paso2 } \\
\hline $\begin{array}{l}\text { Autoeficacia Generalizada } \\
\qquad \begin{array}{c}\text { Paso3 }\end{array}\end{array}$ & $-0,07$ & 0,20 & 0,00 & 1,103 & 0,89 \\
\hline Competencia P. Salud & $0,56 \cdots$ & 0,44 & 0,24 & 1,102 & $430,20^{\cdots *}$ \\
\hline \multicolumn{6}{|l|}{ Satisfacción } \\
\hline \multicolumn{6}{|l|}{ Paso1 } \\
\hline Energía & $0,18^{*}$ & 0,29 & 0,29 & 5,104 & $80,66^{* * *}$ \\
\hline Tesón & $-0,15$ & & & & \\
\hline Estabilidad Emocional & 0,01 & & & & \\
\hline Afabilidad & $0,18^{*}$ & & & & \\
\hline Apertura Mental & 0,03 & & & & \\
\hline $\begin{array}{c}\text { Paso2 } \\
\text { Autoeficacia Generalizada } \\
\text { Paso3 }\end{array}$ & $0,41^{\cdots}$ & 0,43 & 0,14 & 1,103 & $260,18^{\cdots *}$ \\
\hline Competencia P. Salud & $0,24^{* *}$ & 0,48 & 0,05 & 1,102 & $80,70^{* *}$ \\
\hline \multicolumn{6}{|l|}{$\begin{array}{c}\text { Conductas de Prevención } \\
\text { Paso1 }\end{array}$} \\
\hline 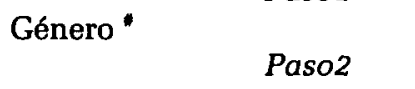 & $0,19^{\star}$ & 0,05 & 0,05 & 1,108 & $50,95^{*}$ \\
\hline Energía & 0,00 & 0,11 & 0,06 & 5,103 & 10,41 \\
\hline Tesón & 0,06 & & & & \\
\hline Estabilidad Emocional & $-0,13$ & & & & \\
\hline Afabilidad & 0,06 & & & & \\
\hline Apertura Mental & $-0,08$ & & & & \\
\hline $\begin{array}{c}\text { Paso3 } \\
\text { Autoeficacia Generalizada } \\
\text { Paso4 }\end{array}$ & $0,34^{* *}$ & 0,21 & 0,10 & 1,102 & $130,86^{\cdots}$ \\
\hline Competencia P. Salud & $0,26^{* *}$ & 0,27 & 0,06 & 1,101 & $70,08^{* *}$ \\
\hline
\end{tabular}

Nota: En los pasos 2, 3 y 4, las $\beta$ y las $R^{2}$ corresponden al modelo global, y el estadístico $F$ al análisis individual.

" 1 = hombre; 2 = mujer.

"**" $p<0,001$; " $p<0,01$; " $p<0,05$. 
Los resultados de estos análisis se muestran en la Tabla 4. En ellos se constata que los rasgos de personalidad explicaron principalmente parte de la varianza del bienestar (20\%) y la satisfacción $(29 \%)$, siendo el porcentaje de varianza menor en el caso de las conductas de prevención (6\%), además de no ser significativo en este último caso el modelo resultante. La AEG dio cuenta también de la variabilidad de la satisfacción (14\%) y las conductas de prevención (10\%), aunque no añadió ninguna validez incremental en la predicción del bienestar. La CPS contribuyó a explicar la variabilidad de los tres criterios de salud, siendo este porcentaje más elevado con respecto al bienestar (24\%), y menor en relación con la satisfacción (5\%) y las conductas de prevención (6\%).

Si se consideran en conjunto los porcentajes de varianza aportados por las dos creencias de autoeficacia, se observa que ambas expectativas, en comparación con los Cinco Factores, dieron cuenta de una mayor variabilidad en el caso del bienestar (24\%) y las conductas de prevención (16\%). En cuanto a la satisfacción, sin embargo, el porcentaje de varianza continuó siendo inferior (19\%) al de los Cinco Factores.

Finalmente se realizaron otra serie de análisis de regresión para determinar la contribución de los Cinco Factores, la AEG y la CPS en la predicción de las estrategias de afrontamiento de los problemas de salud. En todos los análisis se introdujeron los predictores señalados. Además, en la predicción del afrontamiento centrado en la distracción, se controló de nuevo el efecto del género.

Como muestra la Tabla 5, los rasgos de personalidad explicaron un $19 \%$ de la variabilidad en el afrontamiento instru-

Tabla 5. Análisis de regresión jerárquicos prediciendo afrontamiento instrumental y emocional

\begin{tabular}{|c|c|c|c|c|c|}
\hline Criterios & $\beta$ & $R^{2}$ & $\Delta R^{2}$ & $g l$ & $F$ \\
\hline \multicolumn{6}{|l|}{ Instrumental } \\
\hline Paso1 & & & & & \\
\hline Energía & $-0,23^{\star}$ & 0,19 & 0,19 & 5,104 & $40,81^{* *}$ \\
\hline Tesón & $0,35^{* * *}$ & & & & \\
\hline Estabilidad Emocional & $-0,00$ & & & & \\
\hline Afabilidad & $-0,07$ & & & & \\
\hline Apertura Mental & 0,14 & & & & \\
\hline Paso2 & & & & & \\
\hline $\begin{array}{l}\text { Autoeficacia Generalizada } \\
\qquad \mathrm{Paso}\end{array}$ & $-0,00$ & 0,20 & 0,01 & 1,103 & 10,05 \\
\hline Competencia P. Salud & $0,39^{\cdots \cdots}$ & 0,31 & 0,11 & 1,102 & $160,91^{* *}$ \\
\hline \multicolumn{6}{|l|}{ Emocional } \\
\hline Paso1 & & & & & \\
\hline Energía & $-0,11$ & 0,17 & 0,17 & 5,104 & $40,15^{*}$ \\
\hline Tesón & 0,03 & & & & \\
\hline Estabilidad Emocional & $-0,16$ & & & & \\
\hline Afabilidad & 0,01 & & & & \\
\hline Apertura Mental & $-0,02$ & & & & \\
\hline $\begin{array}{c}\text { Paso2 } \\
\text { Autoeficacia Generalizada } \\
\text { Paso3 }\end{array}$ & $-0,18$ & 0,21 & 0,04 & 1,103 & $50,30^{*}$ \\
\hline Competencia P. Salud & $-0,24^{*}$ & 0,25 & 0,04 & 1,102 & $50,91^{\circ}$ \\
\hline
\end{tabular}

Nota: En los pasos 2 y 3 , las $\beta$ y las $R^{2}$ corresponden al modelo global, y el estadístico $F$ al análisis individual. ". $p<0,001 ; " p<0,01 ; " p<0,05$. 
mental, y un $17 \%$ en el afrontamiento emocional. Por otra parte, la AEG apenas dio cuenta de la variabilidad del afrontamiento instrumental ( $1 \%$ ) y el emocional $(4 \%)$, mientras que la CPS añadió una mayor validez incremental en el caso del afrontamiento instrumental (11\%), en comparación con el emocional (4\%).

En cuanto al afrontamiento paliativo y al centrado en la distracción, ninguno de los modelos de regresión resultó significativo, aunque el género (mujer) y la CPS predijeron en sentido positivo el afrontamiento de distracción $(\beta=0,21 ; p<0,05)$ $\mathrm{y}$ el afrontamiento paliativo $(\beta=0,23 ; p$ $<0,05)$, respectivamente.

\section{DISCUSIÓN}

El objetivo central de este trabajo fue explorar y comparar la capacidad predictiva de los Cinco Factores de personalidad, y de la AEG y la CPS, en relación con diversos criterios de bienestar, y con las estrategias de afrontamiento empleadas ante los problemas de salud.

Los resultados obtenidos demostraron, en primer lugar, que los rasgos de personalidad explicaban un porcentaje de la variabilidad de la satisfacción personal más alto que el aportado por las creencias de autoeficacia. En el caso del bienestar, y en contra de lo esperado, el predictor más importante fue la CPS, siendo su contribución superior a la de los rasgos de personalidad. La AEG por su parte no resultó significativa en la predicción de este criterio. No obstante, con respecto a las conductas de prevención, la AEG dio cuenta del mayor porcentaje de varianza, seguida de la CPS. En este caso, los Cinco Factores no resultaron significativos ni explicaron ningún porcentaje de la variabilidad de las conductas preventivas.

El hecho de que la CPS tuviera un peso mayor que las dimensiones básicas de la personalidad en la predicción del bienestar, podría explicarse considerando la similitud en el contenido de los ítems que conforman la escala de CPS, y el de los ítems que se incluyen en la escala de bienestar; siendo más irrelevante en este punto el ajuste en el nivel de generalización de las variables predictoras y la variable criterio. Es decir, la creencia de sentirse capaz de solucionar los problemas relativos a la salud posee una influencia más predominante sobre el bienestar experimentado, que la que podrían ejercer los rasgos de personalidad en su conjunto, y la Extraversión en particular. En este sentido, diferentes investigaciones han puesto de relieve que la CPS mantiene una clara asociación positiva con diferentes aspectos relacionados con la salud y el bienestar emocional, tanto en muestras de pacientes crónicos como en muestras de población sana (Bonetti et al., 2001; Martín-Aragón, Pastor, Lledó et al., 2001; Smith et al., 1995).

Un dato que llama la atención en cuanto a la relación entre el bienestar y la AEG, es la falta de asociación evidenciada entre estas dos variables en el análisis de regresión. Dada la correlación entre la AEG y la CPS $(r=0,72, p<0,001)$, este resultado podría deberse a un efecto de multicolinealidad. Por este motivo, se llevó a cabo un nuevo análisis de regresión sin introducir esta vez la CPS. La AEG resultó entonces significativa $(\beta=0,23$, $p<0,01$, si bien su coeficiente de regresión no fue tan alto como el conseguido por la CPS $(\beta=0,56, p<0,001)$, dato éste que vendría a constatar una cierta independencia en la capacidad predictiva de estas dos expectativas.

Por otra parte, los rasgos de personalidad tuvieron un papel más relevante en la predicción de la satisfacción personal, si bien los principales predictores de la misma fueron la AEG y la CPS. Siguiendo a Diener (1996) la satisfacción con 
uno mismo y con la vida que se lleva podría entenderse como una condición con una mayor estabilidad y consistencia, en vez de como una respuesta afectiva momentánea. Ello explicaría el que los aspectos más estructurales de la personalidad se relacionaran más estrechamente con este criterio.

De igual modo, la satisfacción personal podría conformarse a partir de dominios de diferente índole, lo que le otorgaría un mayor grado de generalización en comparación con el nivel de bienestar. Así por ejemplo, Cooper, Okamura y McNeil (1995) emplearon en su estudio una medida global de satisfacción, en la que se incluían las dimensiones de autonomía, competencia, crecimiento personal, autoaceptación, objetivos vitales y relaciones positivas. El criterio general de satisfacción correlacionó positivamente con el rasgo de extraversión, así como con la actividad social y con el bienestar derivado de esta actividad. Este dato es congruente con los obtenidos en el presente trabajo, ya que, en este caso, dos de las características que predijeron de forma positiva la satisfacción fueron la Energía y la Afabilidad.

En cuanto a las conductas de prevención, la hipótesis formulada fue confirmada al explicar las dos expectativas de nivel intermedio un porcentaje de variabilidad más alto que el aportado por los Cinco Factores. El peso de la AEG fue además superior al de la CPS.

Estos resultados, a la vez que avalan la idea de que la creencia de autoeficacia resulta de gran relevancia en el mantenimiento de un estilo de vida saludable (Bandura, 1997; Gebhardt et al., 2001; Leganger y Kraft, 2003), sugieren que, en la realización de este tipo de conductas, la percepción generalizada de autoeficacia podría tener un efecto superior al de la creencia de competencia relativa a la salud. A este respecto una cuestión interesante para ser examinada en el futuro es la forma y el grado en que esta expectativa más generalizada pudiera estar induciendo, ante cada conducta de salud, creencias específicas de autoeficacia.

Asimismo, puesto que ninguna de las dimensiones básicas de la personalidad resultó predictora de las conductas de prevención, también se estaría apoyando la idea ampliamente mantenida en la literatura sobre la necesidad de incorporar variables más contextuales y dinámicas en la predicción de criterios estrechamente vinculados con la salud (Bermúdez, 1999; Booth-Kewley y Vikers, 1994; Conner y Abraham, 2001; Marshall et al., 1994).

De otro lado los resultados del presente estudio revelaron que, con respecto al afrontamiento instrumental y emocional de los problemas de salud, los Cinco Factores dieron cuenta de un mayor porcentaje de variabilidad, en comparación con las expectativas de nivel intermedio. Además, mientras que la CPS predijo de forma significativa ambos tipos de afrontamiento, la AEG no alcanzó en ningún caso el nivel de significación. En relación con el afrontamiento paliativo y el centrado en la distracción, ni las dimensiones básicas de personalidad ni las creencias de autoeficacia contribuyeron a explicar su variabilidad.

Estos resultados son interesantes en la medida en que, hasta la fecha, no se ha encontrado ninguna otra investigación que aborde conjuntamente las relaciones de los Cinco Factores y las creencias de autoeficacia con respecto a las respuestas de afrontamiento puestas en marcha ante los problemas de salud.

El afrontamiento instrumental fue predicho positivamente por el rasgo de Tesón y la CPS, aspecto éste que avala la evidencia hallada en otros trabajos (Bouchard, Guillemette y Landry-Léger, 2004; Penley y Tomaka, 2002; Rueda, 2003). De esta manera, características como la orientación hacia el logro y la tendencia 
hacia la planificación de las tareas, así como la estimación de ser capaz de manejar los asuntos de la salud eficazmente, se asociarían con un tipo de afrontamiento de las enfermedades más activo, dirigido principalmente a buscar información y ayuda médica.,

En relación con el afrontamiento emocional hay que indicar que, si bien los Cinco Factores explicaron un porcentaje de varianza superior al de las creencias de autoeficacia, ninguno de ellos resultó ser un predictor significativo. La CPS, sin embargo, sí predijo en sentido negativo este tipo de afrontamiento, poniendo así de manifiesto que el mantenimiento de esta creencia, además de promover la solución de los problemas de salud, amortigua el uso de estrategias dirigidas a una regulación inefectiva de las emociones, actuación ésta que, a su vez, podría favorecer un manejo más apropiado de la enfermedad.

Finalmente cabe destacar que, ni las dimensiones básicas de la personalidad ni las expectativas de autoeficacia, dieron cuenta de la variabilidad del afrontamiento paliativo y el dirigido a la distracción. En este sentido se podría especular que estas respuestas de afrontamiento podrían ser menos disposicionales que el afrontamiento instrumental o emocional, iniciándose, por tanto, en fases concretas del proceso de enfermedad, o sólo ante problemas de salud determinados. Esta explicación, meramente aproximativa, requiere la obtención de una mayor evidencia empírica, a través de la cual se pueda esclarecer el papel de estos dos tipos de afrontamiento ante diferentes problemas de salud.

La presente investigación cuenta con ciertas limitaciones, que deben ser tenidas en cuenta para una interpretación más exacta de los resultados comentados. Por una parte hay que tener presente que todas las medidas empleadas en este trabajo fueron autoinformadas, lo que ha podido incrementar la magnitud de las correlaciones obtenidas. Por ello sería necesario replicar este trabajo recurriendo a criterios de autoeficacia, afrontamiento y salud menos sesgados por las valoraciones subjetivas.

De otra parte destaca la naturaleza correlacional del estudio, lo cual impide el establecimiento de relaciones causales entre las variables. De ahí que no pueda descartarse la posible influencia del estado de bienestar o la satisfacción sobre la creencia de autoeficacia y la percepción de competencia en salud, generándose así asociaciones bidireccionales.

Asimismo una variable que no fue controlada en la investigación, y que parece tener un claro efecto sobre la competencia percibida en salud y el bienestar, es el estado de salud (Smith et al., 1995). Al estar compuesta la muestra, en su mayoría, por participantes jóvenes y estudiantes, es posible que su experiencia con la enfermedad fuera más reducida, siendo así su nivel de competencia percibida en salud y de bienestar más altos. Este aspecto pone de manifiesto la necesidad de llevar a cabo nuevos trabajos con otro tipo de poblaciones, para poder alcanzar una mayor generalización de los resultados.

Por último queda abierto el camino para abordar los objetivos planteados en este estudio desde una óptica más específica, atendiendo a las creencias de autoeficacia y a las estrategias de afrontamiento que se inician ante determinadas enfermedades, así como a la realización y mantenimiento de hábitos concretos de salud.

A pesar de estas limitaciones, el presente estudio ha resaltado la importancia de examinar conjuntamente la utilidad predictiva de las dimensiones básicas de la personalidad y de los factores más contextuales y dinámicos, para poder comprender con mayor rigor el funciona- 
miento de todas estas variables dentro del ámbito del bienestar y la prevención. Asimismo esta investigación ha proporcionado una mayor evidencia respecto de las relaciones entre la personalidad y la forma de afrontar los problemas de salud.

\section{REFERENCIAS BIBLIOGRÁFICAS}

Baessler, J., y Schwarzer, R. (1996). Evaluación de la autoeficacia: adaptación española de la escala de autoeficacia general. Ansiedad y Estrés, 2, 1-8.

Bandura, A. (1977). Self-efficacy: Toward a unifying theory of behavioural change. Psychological Review, 84, 191-215.

Bandura, A. (1989). Human agency in social cognitive theory. American Psychologist, $44,1175-1184$.

Bandura, A. (1997). Self-efficacy: The exercise of control. New York: W.H. Freeman and Company.

Barbaranelli, C., y Caprara, V.G. (2002). Studies of the Big Five Questionnaire. En B. de Raad y M. Perugini (Eds.), Big Five Assessment (pp.108-128). Seattle: Hogrefe and Huber Publishers.

Bermúdez, J. (1999). Personality and healthprotective behaviour. European Journal of Personality, 13, 83-103.

Bonetti, D., Johnston, M., Rodríguez-Marín, J., Pastor, M.A., Martín-Aragón, M., Doherty, E., y Sheehan, K. (2001). Dimensions of perceived control: $A$ factor analysis of three measures and an examination of their relation to activity level and mood in a student and cross-cultural patient sample. Psychology and Health, 16, 655-674.

Booth-Kewley S., y Vickers, R.R.Jr. (1994). Associations between major domains of personality and health behavior. Journal of Personality, 60, 281-298.

Bouchard, G., Guillemette, A., y LandryLéger, N. (2004). Situational and dispositional coping: An examination of their relation to personality, cognitive appraisals, and psychological distress. European Journal of Personality, 18, 221-238.

Cantor, N. (1990). From thought to behaviour: "Having" and "Doing" in the study of per- sonality and cognition. American Psychologist, 45, 735-750.

Caprara, G.V., Barbaranelli, C., y Borgogni, L. (1995). BFQ-Cuestionario «Big-Five». Madrid: TEA Ediciones. (Adaptación española de J. Bermúdez).

Caprara, G.V., Barbaranelli, C., Borgogni, L., y Perugini, M. (1993). The «Big Five Questionnaire»: A new questionnaire to assess the five factor model. Personality and Individual Differences, 15, 281-288.

Carver, C.S., Scheier, M.F., y Weintraub, J.K. (1989). Assessing coping strategies: A theoretically based approach. Journal of Personality and Social Psychology, 56, 267283.

Conner, M., y Abraham, C. (2001). Conscientiousness and the Theory of Planned Behavior: Toward a more complete model of antecedents of intentions and behavior. Personality and Social Psychology Bulletin, 27, 1547-1561.

Cooper, H., Okamura, L., y McNeil, P. (1995). Situation and personality correlates of psychological well-being: Social activity and personal control. Journal of Research in Personality, 29, 305-417.

Chang, E.C. (1998). Dispositional optimism and primary and secondary appraisal of a stressor: Controlling for confounding influences and relations to coping and psychological and physical adjustment. Journal of Personality and Social Psychology, 74, 1109-1120.

DeNeve, K.M., y Cooper, H. (1998). The happy personality: A meta-analysis of $\mathbf{1 3 7}$ personality traits and subjective wellbeing. Psychological Bulletin, 124, 197229.

Diener, E. (1996). Traits can be powerful, but are not enough: Lessons from subjective well-being. Journal of Research in Personality, 30, 389-399.

Emmons, R.A., y Diener, E. (1985). Personality of correlates of subjective well-being. Personality and Social Psychology Bulletin, 11, 89-97.

Endler, N.S., y Parker, J.D.A. (1999). Coping with Health Injuries and Problems (CHIP): Manual. Toronto: Multi-Health Systems. Endler, N.S., Courbasson, C.M.A., y Fillion, L. (1998). Coping with cancer: The eviden- 
ce for the temporal stability of the FrenchCanadian version of the coping with health injuries and problem (CHIP). Personality and Individual Differences, 25, 711-717.

Endler, N.S., Kocovski, S., y Macrodimitris, S.D. (2001). Coping, efficacy, and perceived control in acute vs. chronic illness. Personality and Individual Differences, 30, 617-625.

Endler, N.S., Parker, J.D.A., y Summerfeldt, L.J. (1993). Coping with health problems: Conceptual and methodological issues. Canadian Journal of Behavioural Science, 25, 384-399.

Endler, N.S., Parker, J.D.A., y Summerfeldt, L.J. (1998). Coping with health problems: Developing a reliable and valid multidimensional measure. Psychological Assessment, 10, 195-205.

Ferguson, E. (2001). Personality and coping traits: A joint factor analysis. British Journal of Health Psychology, 6, 311-325.

Folkman, S. (1984). Personal control and stress and coping processes. A theoretical analysis. Journal of Personality and Social Psychology, 46, 839-852.

Gebhardt, W.A., van der Doef, M.P., y Paul, L.B. (2001). The revised health hardiness inventory (RHHI-24): Psychometric properties and relationship with self-reported health and health behavior in two Dutch samples. Health Education Research, 16, 579-592.

John, O.P. (1990). The «Big Five» factor taxonomy: Dimensions of personality in the natural language and in questionnaires. En L.A. Pervin (Ed.), Handbook of Personality: Theory and Research (pp. 66-100). New York: The Guilford Press.

Judge, T.A., Erez, A., Bono, J.E., y Thoresen, C.J. (2002). Are measures of self-esteem, neuroticism, locus of control, and generalized self-efficacy indicators of a common core construct? Journal of Personality and Social Psychology, 83, 693-710.

Lazarus, R.S., y Folkman, S. (1987). Transactional theory and research on emotions and coping. European Journal of Personality, 1, 141-169.

Legander, A., y Kraft, P. (2003). Control constructs: Do they mediate the relation between educational attainment and health behaviour? Journal of Health Psychology, 8, 361-372.

Lemos-Giráldez, S., y Fidalgo-Aliste, A. (1997). Personality dispositions and health-related habits and attitudes: A cross-sectional study. European Journal of Personality, 11, 109-209.

Marks, G.R., y Lutgendorf, S.K. (1999). Perceived Health Competence and Personality Factors differentially predict health behaviors in older adults. Journal of Aging and Health, 11, 221-239.

Marshall, G.N., Wortman, C.B., Vickers, R.R. Jr., Kusulas, J.W., y Hervig, L.K. (1994). The five-factor model of personality as a framework for personality-health research. Journal of Personality and Social Psychology, 67, 278-286.

Martín-Aragón, M., Pastor, M.A., Lledó, S., López-Roig, M.C., Terol, M.C., y Rodríguez-Marín, J. (2001). Percepción de control en el síndrome fibromiálgico: variables relacionadas. Psicothema, 13, 586-591.

McCrae, R.R., y Costa, P.T. Jr. (1985). Updating Norman's adequate taxonomy: Intelligence and personality dimensions in natural language and in questionnaires. Journal of Personality and Social Psychology, 49, 710-721.

McCrae, R.R., y Costa, P.T. Jr. (1986). Personality, coping, and coping effectiveness in an adult sample. Journal of Personality, 54, 385-405.

McCrae, R.R., y Costa, P.T. Jr. (1987). Validation of the Five-Factor model of personality across instruments and observers. Journal of Personality and Social Psychology, 52, 81-90.

McCrae, R.R., y Costa, P.T. Jr. (1991). Adding liebe und arbeit: The full Five-Factor model and well-being. Personality and Social Psychology Bulletin, 17, 227-232.

McCrae, R.R., y Costa, P.T. Jr. (1997). Personality trait structure as a human universal. American Psychologist, 52, 509-516.

Norman, W.T. (1963). Toward an adequate taxonomy of personality attributes: Replicated factors structure in peer nomination personality ratings. Journal of Abnormal and Social Psychology, 66, 574-583.

Pastor. M.A., López-Roig, S., RodríguezMarín, J., Martín-Aragón, M., Terol, M.C., 
y Pons, N. (1999). Percepción de control, impacto de la enfermedad y ajuste emocional en enfermos crónicos. Ansiedad y Estrés, 5, 299-311.

Pavot, W., Diener, E., y Fujita, F. (1990). Extraversion and happiness. Personality and Individual Differences, 11, 1299-1306.

Penley, J.A., y Tomaka, J. (2002). Associations among the Big Five, emotional responses, and coping with acute stress. Personality and Individual Differences, 32, 1215-1228.

Prenda, K.M., y Lachman, M.E. (2001). Planning for the future: A life management strategy for increasing control and life satisfaction in adulthood. Psychology and Aging, 16, 206-216.

Rueda, B. (2003). Competencia percibida y salud: aproximación general y aplicación a la enfermedad cardiovascular. Tesis doctoral no publicada. Madrid: UNED.

Rueda, B., Pérez-García, A.M., y Bermúdez, J. (2003). La salud emocional desde la perspectiva de la competencia percibida. Acción Psicológica, 2, 41-49.

Ruiz, M.A., y Baca, E. (1993). Design and validation of the "Quality of Life Questionnaire": A generic health-related Quality of Life Instrument. European Journal of Psychological Assessment, 9, 19-32.

Sanjuán, P., Pérez-García, A.M., y Bermúdez, J. (2000). Escala de autoeficacia general: datos psicométricos de la adaptación para población española. Psicothema, 12, 509513.

Sanz, A., y Villamarín, F. (1999). Autoeficacia, valor del incentivo y competencia personal: efecto sobre el estado de ánimo, la motivación intrínseca y la activación percibida. Ansiedad y Estrés, 5, 145-160.

Schwarzer, R. (1993). Measurement of perceived self-efficacy. Psychometric scales for cross-cultural research. Berlin: Freie Universität.
Schwarzer, R. (1999). Self-regulatory processes in the adoption and maintenance of health behaviors. Journal of Health Psychology, 4, 115-127.

Smith, M.S., Wallston, K.A., y Smith, C.A. (1995). The development and validation of the Perceived Health Competence Scale. Health Education Research, 10, 51-64.

Smith, T.W., y Williams, P.G. (1992). Personality and health: Advantages and limitations of the five-factor model. Journal of Personality, 60, 395-423.

Van Heck, G.L. (1997). Personality and physical health: Toward an ecological approach to health-related personality research. European Journal of Personality, 11, 415-443.

Vollrath, M., y Torgersen, S. (2002). Who takes health risks? A probe into eight personality types. Personality and Individual Differences, 32, 1185-1197.

Vollrath, M., Knoch, D., y Cassano, L. (1999). Personality, risky health behaviour, and perceived susceptibility to health risks. European Journal of Personality, 13, 39-50.

Wallston, K.A. (1992). Hocus-pocus, the focus is not strictly on locus: Rotter's social learning theory modified for health. Cognitive Therapy and Research, 16, 183-199.

Wasylkiw, L., y Fekken, C.G. (2002). Personality and self-reported health: Matching predictors and criteria. Personality and Individual Differences, 33, 607-620.

Watson, D., y Clark, L.A. (1992). Affects separable and inseparable: On the hierarchical arrangement of the negative affects. Journal of Personality and Social Psychology, 62, 489-505.

Watson, D., y Hubbard, B. (1996). Adaptational style and dispositional structure: Coping in the context of the Five - Factor Model. Journal of Personality, 64, 737-773.

Weinstein, N.D. (1993). Testing four competing theories of Health-Protective Behavior. Health Psychology, 12, 324-333. 